\title{
Rinite ocupacional - Desafios na abordagem diagnóstica e terapêutica
}

\section{Occupational rhinitis - Diagnostic and therapeutic challenges caregivers}

Data de receção / Received in: 25/9/2020

Data de aceitação / Accepted for publication in: I/ I I/2020

\begin{abstract}
Rev Port Imunoalergologia 202I; 29 (2): 107-1 I9
\end{abstract}

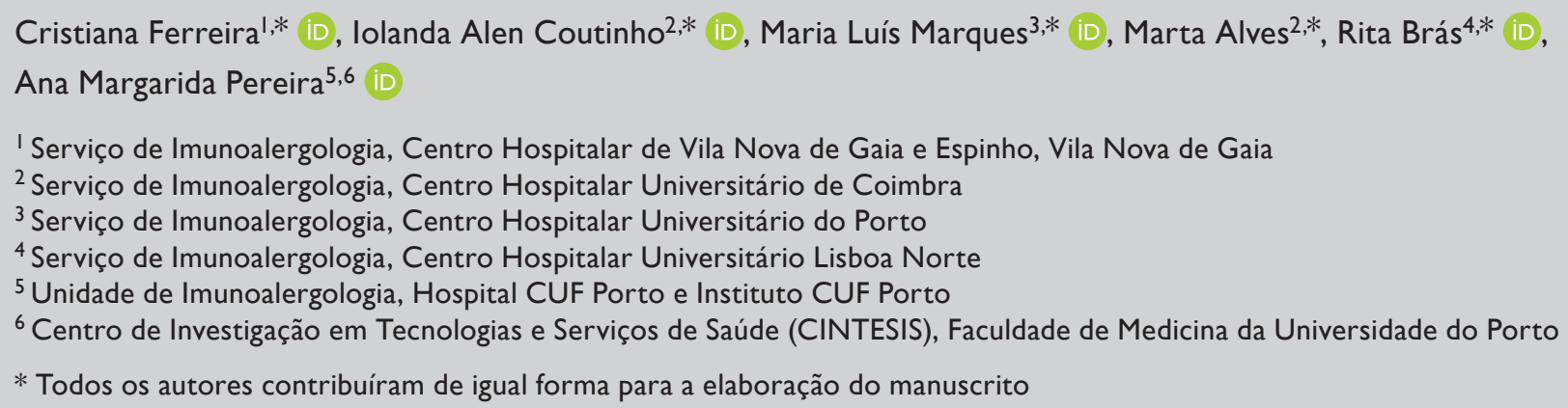

\section{RESUMO}

A rinite ocupacional é uma patologia inflamatória da mucosa nasal caracterizada por sintomas nasais intermitentes ou persistentes e/ou limitação variável do fluxo aéreo nasal e/ou hipersecreção nasal devido a causas atribuíveis a um ambiente de trabalho específico. Pode ser classificada em alérgica (lgE-mediada ou não lgE-mediada) ou não alérgica. A rinite ocupacional alérgica é habitualmente a expressão clínica inaugural da sensibilização das vias aéreas a um alergénio presente no ambiente laboral e pode preceder o aparecimento da asma ocupacional. Embora o diagnóstico seja essencialmente clínico, pelos aspetos médico-legais envolvidos, torna-se importante identificar os agentes etiológicos, sendo a prova de provocação nasal o gold-standard diagnóstico; no entanto, quando não está disponível, poderá ser feita uma avaliação no local de trabalho. A opção terapêutica mais eficaz consiste na evicção da exposição. Neste artigo reunimos a evidência científica atual relativa à rinite ocupacional, partindo de um caso clínico para contextualização do problema.

Palavras-chave: Diagnóstico, rinite ocupacional, tratamento. 


\section{ABSTRACT}

Occupational rhinitis is an inflammatory disease of the nasal mucosa, characterized by intermittent or persistent nasal symptoms and/or variable limitation of nasal airflow and/or nasal hypersecretion due to causes attributable to a specific work environment. It can be classified as allergic (IgE-mediated or non-lgE-mediated) or non-allergic. Allergic occupational rhinitis is usually the inaugural clinical expression of airway sensitization to an allergen present in the workplace and may precede the onset of occupational asthma. Although the diagnosis is essentially clinical, due to the medical-legal aspects involved, it is important to identify the etiological agents, being the nasal provocation test considered the gold-standard of diagnosis; however, when not available, a workplace assessment can be made. The most effective therapeutic option is to avoid exposure. In this article we gather the current scientific evidence related to occupational rhinitis, starting from a clinical case to contextualize the problem.

Keywords: Diagnosis, occupational rhinitis, treatment.

(C) 202I Sociedade Portuguesa de Alergologia e Imunologia Clínica. Published by Publicações Ciência \& Vida. This is an open access article under the CC BY-NC-ND license (http://creativecommons.org/licenses/by-nc-nd/4.0/).

$\mathrm{E}$ ste artigo começa com um caso clínico sumário ilustrativo de um problema clínico frequente. Posteriormente, é apresentada a evidência relativa a essa patologia, focando diferentes aspetos que se consideram clinicamente relevantes, incluindo formas de abordagem. $\mathrm{O}$ artigo termina com as recomendações clínicas dos autores, tendo por base o caso clínico inicial.

\section{CASO CLÍNICO}

Homem de 54 anos, com história de obstrução nasal bilateral, rinorreia anterior, crises esternutatórias, prurido nasal, hiperemia conjuntival e lacrimejo com 10 anos de evolução. Nos últimos dois anos, também com tosse e sensação de dispneia aquando do agravamento do quadro nasal. Trabalha como pasteleiro desde há 30 anos e associa as queixas exclusivamente ao contacto com farinhas no local de trabalho, negando sintomas no domicílio e durante o período de férias. A rinoscopia anterior revelou uma mucosa empalidecida e ligeira hipertrofia dos cornetos inferiores e a auscultação pul- monar murmúrio vesicular audível bilateralmente, sem ruídos adventícios.

\section{INTRODUÇÃO}

A rinite é definida como uma inflamação da mucosa nasal, caraterizada por sintomas nasais, como limitação do fluxo aéreo nasal, rinorreia anterior e/ou posterior, prurido nasal e espirros'.

A rinite relacionada com o trabalho engloba duas formas de apresentação: rinite ocupacional (RO) e rinite exacerbada pelo trabalho².

A rinite exacerbada pelo trabalho ocorre em doentes com rinite preexistente que apresentam agravamento clínico com a exposição ambiental no local de trabalho ${ }^{3}$.

A European Academy of Allergy and Clinical Immunology (EAACI) define RO como "uma doença inflamatória do nariz caracterizada por sintomas intermitentes ou persistentes (ou seja, congestão nasal, espirros, rinorreia e prurido) e/ou limitação variável do fluxo aéreo nasal e/ou hipersecreção nasal devido a causas e condições atribuí- 
veis a um ambiente de trabalho específico e não a estímulos encontrados fora do local de trabalho". A RO pode ainda ser subdividida em alérgica e não alérgica ${ }^{4}$.

A RO alérgica é habitualmente a expressão clínica inaugural da sensibilização das vias aéreas a um alergénio existente no ambiente de trabalho ${ }^{5}$. Caracteriza-se clinicamente pelo desenvolvimento de hipersensibilidade a um agente ocupacional específico, após um período de latência necessário para adquirir sensibilização imunológica ao agente causal, resultando em sintomas reprodutíveis, intermitentes ou persistentes, de acordo com a frequência e intensidade com que ocorrem ${ }^{4}$. Imunologicamente pode ser mediada por lgE, resultando habitualmente da exposição a agentes de alto peso molecular, ou seja, glicoproteínas de origem vegetal e animal, e algumas substâncias de baixo peso molecular, como os sais de platina, reativos, corantes e anidridos ácidos. A RO alérgica não mediada por lgE pode ser induzida por agentes de baixo peso molecular atuando como haptenos, em que o mecanismo alérgico ainda não foi totalmente caraterizado.

Vários estudos demonstraram que cerca de $15 \%$ dos trabalhadores têm RO, o que representa $4 \%$ das doenças respiratórias ocupacionais ${ }^{6}$. $O$ tipo e reatividade dos alergénios, condições de trabalho, tipo de técnicas e processos industriais, idade e atopia são exemplos de fatores de risco que parecem afetar a prevalência de $\mathrm{RO}^{6}$.

A RO não alérgica resulta do contacto não imunológico de irritantes (vapores, fumo, pó...) do ambiente de trabalho com a mucosa nasal. Diferentes exposições ocupacionais têm sido associadas a sintomas de rinite, frequentemente com um padrão histológico predominantemente neutrofílico. Os sintomas podem ocorrer apenas durante a exposição a irritantes ou podem ser persistentes ${ }^{4,7,8}$.

A RO pode ter um impacto negativo importante na qualidade de vida do doente, resultando em dificuldades no desempenho laboral, produtividade reduzida e problemas psicossociais. A RO pode ainda coexistir com asma ocupacional (AO), existindo evidência a favor da relação entre a gravidade da RO e a gravidade da asma ${ }^{3,9}$.

\section{EPIDEMIOLOGIA}

As doenças alérgicas ocupacionais são comuns e a sua prevalência parece estar a aumentar ${ }^{10}$. Embora a prevalência da asma ocupacional tenha sido amplamente estudada, a epidemiologia da rinite ocupacional tem sido menos investigada, o que em parte pode ser explicado pelo facto de não ser considerada uma doença grave ${ }^{\prime \prime}$.

A prevalência da rinite ocupacional em doentes com asma ocupacional tem sido reportada em 76-92\% ${ }^{11,12}$. De facto, tem sido reconhecido que a rinite ocupacional coexiste com a asma ocupacional ${ }^{11,13}$, precedendo-a habitualmente ${ }^{14}$. No entanto, são escassos os estudos que avaliaram a relação entre o início da rinite ocupacional e a asma ocupacional. Tal como a prevalência da rinite é maior do que a prevalência da asma na população mundial ${ }^{15}$, estima-se que a prevalência da asma ocupacional seja de 5 a $15 \%^{10}$ e a da rinite ocupacional parece ser duas a quatro vezes superior ${ }^{16-19}$.

As estimativas da prevalência da rinite ocupacional variam de acordo com os métodos utilizados no diagnóstico (questionários, avaliação objetiva), a atividade ocupacional estudada e a área geográfica em questão, limitando a comparação entre os vários estudos de prevalência que se encontram publicados. Assim, a prevalência pode estar subestimada devido aos seguintes fatores: I) o efeito do trabalhador saudável, que é uma fonte de viés relacionada com o facto de indivíduos mais doentes escolherem ambientes de trabalho em que as exposições são menores ou poderem ser excluídos da contratação laboral ${ }^{20}$; 2) trabalhadores já contratados poderem solicitar a transferência para setores de atividade com menor exposição ou abandonar o posto de trabalho' ${ }^{21}$;) a relutância dos trabalhadores em relatar sintomas por receio de perder o emprego 22 .

Embora a prevalência global de rinite ocupacional seja desconhecida, estão identificadas profissões com maior risco: trabalhadores da indústria das peles, têxtil, das madeiras, de processamento de alimentos, manipuladores de químicos, plásticos e borrachas, metalúrgicos, operá- 
rios de montagem de produtos elétricos e eletrónicos, pintores à pistola, padeiros, agricultores, técnicos de laboratório, dentistas e veterinários ${ }^{4,23,24}$. $O$ risco de rinite ocupacional em várias profissões foi avaliado num estudo que analisou 1244 casos diagnosticados de rinite ocupacional relatados de 1986 a 1991 no Registo Finlandês de Doenças Profissionais. $O$ maior risco de rinite ocupacional foi entre os trabalhadores da indústria das peles, seguidos pelos padeiros, criadores de gado, trabaIhadores de processamento de alimentos, veterinários, agricultores, operários de montagem de produtos elétricos, eletrónicos e de telecomunicações e construtores de barcos ${ }^{25}$. Um estudo posterior identificou os médicos e profissionais de farmácia como grupos adicionais de alto risco ${ }^{24}$. Têm sido reportadas prevalências díspares de rinite ocupacional em determinados grupos profissionais expostos a agentes específicos, provavelmente devido a condições de exposição diferentes e aos hábitos socioeconómicos próprios de cada país.

A rinite ocupacional e a asma ocupacional partilham agentes etiológicos e mecanismos patogénicos ${ }^{11,26,27}$. Assim, os agentes etiológicos da rinite ocupacional (Quadro I), à semelhança da asma ocupacional, classificam-se, de acordo com o peso molecular, em partículas de alto peso molecular (superior a $5 \mathrm{kDa}$ ) (APM) e partículas de baixo peso molecular (inferior a $5 \mathrm{kDa}$ ) (BPM) ${ }^{19,28}$. Os principais alergénios na rinite alérgica ocupacional são partículas APM, em geral glicoproteínas de origem animal ou vegetal e enzimas, que induzem tipicamente uma resposta alérgica IgE-mediada: epitélios de animais, ácaros de armazenamento, fungos, gomas vegetais, látex, farinhas de cereais, café, algodão, alfa-amilase e outras enzimas biológicas ${ }^{4,29}$. A rinite não alérgica ocupacional é, na maioria dos casos, induzida pela exposição a partículas BPM, que são substâncias inorgânicas que podem funcionar como haptenos; salientam-se, di-isocianatos, anidridos, sais metálicos, persulfatos, fármacos, desinfetantes, tintas, formaldeído e acrilatos ${ }^{30}$. A prevalência de rinite ocupacional variou entre 2 e $87 \%$ nos trabalhadores expostos a agentes APM e entre 3 e $48 \%$ nos expostos a agentes
$\mathrm{BPM}^{\prime \prime}$. Atualmente admite-se de uma forma consensual que a rinite ocupacional é um "marcador" precoce da asma ocupacional $1^{5,11}$. Os doentes com rinite ocupacional constituem assim um grupo-alvo particularmente relevante para implementar medidas de prevenção.

Relativamente aos fatores de risco para rinite ocupacional, a exposição, a atopia e o tabagismo parecem constituir os principais determinantes". Um gradiente de dose-resposta entre o nível de exposição e a sensibilização IgE-mediada foi substanciado para vários agentes APM, como animais de laboratório, farinhas, insetos, alfa-amilase e enzimas de detergentes ${ }^{4}$. No entanto, a maioria dos estudos relacionam-se mais fortemente com a sensibilização do que com a clínica de rinite ocupacional" . A atopia tem sido associada a um risco aumentado de sensibilização a agentes APM e a rinite ocupacional causada por estes compostos ${ }^{16}$. Os estudos disponíveis forneceram resultados inconsistentes no que diz respeito à relação entre atopia e rinite ocupacional em trabalhadores expostos a agentes BPM ${ }^{11,31}$.

Ao contrário do que acontece com asma ocupacional, o papel dos fatores genéticos no desenvolvimento de rinite ocupacional não foi especificamente avaliado ${ }^{32}$. O tabagismo tem sido assumido como fator de risco para doenças ocupacionais, no entanto, o efeito do tabagismo na rinite ocupacional não se encontra totalmente esclarecido. De facto, as relações entre tabagismo, rinite ocupacional e asma ocupacional permanecem controversas: o tabagismo não foi estabelecido como fator de risco para a rinite ocupacional, embora aumente o risco de desenvolver algumas formas de asma ocupacional ${ }^{33,34}$. No que respeita à asma ocupacional, estão bem definidos os fatores de risco: a exposição é um fator causal, a atopia e a predisposição genética são fatores predisponentes e o tabagismo é um fator contributivo. Já em relação à rinite ocupacional, a exposição parece ser o fator de risco mais relevante; no entanto, não está totalmente esclarecido o seu contributo, pois a exposição correlaciona-se com a sensibilização, mas nem sempre se correlaciona diretamente com o aparecimento de sintomas de rinite ocupacional ${ }^{25,32}$. 
Quadro I. Principais agentes etiológicos e profissões implicadas na rinite ocupacional ${ }^{28,60}$

\begin{tabular}{|c|c|}
\hline Agentes & Profissão \\
\hline \multicolumn{2}{|r|}{ Baixo peso molecular } \\
\hline Isocianatos & Pintores à pistola, operários na produção de poliuretano/plástico e fundições \\
\hline Anidridos & $\begin{array}{l}\text { Operários em indústrias de produção de resinas epóxi, vernizes, tintas, plásticos, } \\
\text { material elétrico/eletrónico }\end{array}$ \\
\hline Poeiras/fibras de algodão & Operários da indústria têxtil \\
\hline $\begin{array}{l}\text { Metais (crómio, níquel, platina, cobalto, } \\
\text { vanádio) }\end{array}$ & $\begin{array}{l}\text { Operários de refinarias, indústria química, metalurgia, metalomecânica, de material } \\
\text { elétrico/eletrónico, soldadores }\end{array}$ \\
\hline $\begin{array}{l}\text { Fármacos (beta-lactâmicos, macrólidos, } \\
\text { isoniazida, opiáceos, citostáticos) }\end{array}$ & $\begin{array}{l}\text { Operários da indústria farmacêutica, profissionais de saúde, trabalhadores em } \\
\text { explorações de agropecuária }\end{array}$ \\
\hline Madeiras & Madeireiros, carpinteiros, operários de indústrias de mobiliário \\
\hline $\begin{array}{l}\text { Corantes e descolorantes } \\
\text { (antraquinona, carmina, extrato de hena, } \\
\text { persulfatos, corantes reativos) }\end{array}$ & Operários da indústria de tinturaria de tecidos e peles, cabeleireiros \\
\hline Aminas (cloramina, aminas quaternárias) & Químicos, trabalhadores de limpeza, fabricantes de plásticos \\
\hline Colas e resinas (acrilatos, resinas epóxi) & Fabricantes de plásticos \\
\hline $\begin{array}{l}\text { Miscelânea (formaldeído, glutaraldeído, } \\
\text { óxido de etileno, piretrina, vapor de cloreto } \\
\text { de polivinilo) }\end{array}$ & $\begin{array}{l}\text { Técnicos de laboratório, trabalhadores de fábricas têxteis, pintores à pistola, } \\
\text { profissionais de saúde }\end{array}$ \\
\hline \multicolumn{2}{|r|}{ Alto peso molecular } \\
\hline Epitélios animais & $\begin{array}{l}\text { Agricultores, veterinários, trabalhadores de explorações de agropecuária, técnicos } \\
\text { de laboratório }\end{array}$ \\
\hline Ácaros & $\begin{array}{l}\text { Técnicos de laboratório, trabalhadores de produção agropecuária, jardinagem e } \\
\text { hortofloricultura, operários da indústria alimentar, trabalhadores em celeiros e silos }\end{array}$ \\
\hline Farinhas e cereais & $\begin{array}{l}\text { Padeiros, pasteleiros, operários da indústria de panificação, trabalhadores em } \\
\text { celeiros, silos e explorações de agropecuária }\end{array}$ \\
\hline $\begin{array}{l}\text { Enzimas (papaína, alfa-amilase, lactase, } \\
\text { protease, lipase, celulase, carboxidase, } \\
\text { tripsina, bromelaína, pectinase) }\end{array}$ & $\begin{array}{l}\text { Padeiros, pasteleiros, operários da indústria de panificação, detergentes, } \\
\text { farmacêutica e plásticos }\end{array}$ \\
\hline Látex & $\begin{array}{l}\text { Profissionais de saúde, operários da produção de luvas de látex, material médico- } \\
\text {-cirúrgico, preservativos, componentes para calçado (solas) }\end{array}$ \\
\hline Peixes e mariscos & Trabalhadores da indústria alimentar \\
\hline Gomas vegetais & Trabalhadores da indústria alimentar, fabrico de carpetes, material elétrico/eletrónico \\
\hline
\end{tabular}

\section{DIAGNÓSTICO}

O diagnóstico de RO implica a demonstração da presença de rinite e da sua relação com a atividade laboral ${ }^{4,35}$. A investigação deve incluir história clínica, exame nasal, testes imunológicos e prova de provocação nasal (PPN), sempre que possível.

\section{História clínica}

A história clínica deve reunir uma descrição completa das tarefas do trabalhador, uso de equipamento de proteção individual, mudanças recentes nos materiais ou processos de trabalho e condições de higiene no local de trabalho. Devem ser requisitadas fichas de dados de segurança dos compostos aos quais o trabalhador está di- 
reta e indiretamente exposto ${ }^{4,35}$. A análise da relação temporal entre o aparecimento dos sintomas e o início do trabalho é crucial para o diagnóstico, bem como a melhoria em períodos fora do trabalho, por exemplo fins-de-semana e férias, e a recorrência de sintomas após a reexposição $4,35,36$.

Deve procurar-se avaliar também a gravidade dos sintomas e o impacto na qualidade de vida do doente ${ }^{4}$.

Os sintomas da RO são semelhantes aos da rinite não ocupacional (esternutos, rinorreia aquosa, obstrução nasal) ${ }^{4,35,36}$. Na RO IgE mediada o prurido nasal também é frequente ${ }^{36}$. Aos sintomas nasais podem também associar-se queixas conjuntivais ou até queixas adicionais de pressão facial, rinorreia posterior e hiposmia, sugerindo progressão para um quadro de rinossinusite. $\mathrm{Na}$ RO alérgica habitualmente verifica-se uma latência dos sintomas de I a 5 anos relativamente ao início do trabaIho, enquanto na RO não alérgica os sintomas podem surgir logo após a primeira exposição ${ }^{4,36}$. Uma vez estabelecida a doença, os sintomas surgem nos primeiros minutos a uma hora após a exposição ao agente causa ${ }^{35}$.

$A$ associação entre $R O$ e $A O$ também deve ser considerada na avaliação inicial do doente. Os indivíduos com RO têm maior risco de desenvolver $A O$, comparando com indivíduos com outras doenças ocupacionais ${ }^{37}$, e há estudos que sugerem que esta tendência é maior na sensibilização a agentes de APM $^{9,12,38}$.

Para além do diagnóstico diferencial com outros tipos de rinite, torna-se importante distinguir a RO da rinite exacerbada pelo trabalho. A última consiste na presença de rinite preexistente ou simultânea, alérgica ou não alérgica, agravada (e não provocada, como acontece na RO) pelas exposições no local de trabalho. Os mecanismos envolvidos ainda não são claros. A sintomatologia poderá ser desencadeada por uma ampla variedade de estímulos presentes no local de trabalho, incluindo agentes irritantes (por exemplo, produtos químicos, pós, vapores), físicos (como as mudanças de temperatura), odores fortes (por exemplo, perfumes), fumo de tabaco ou até emoções. A resposta nasal pode ser afetada pela idade, sexo e/ou presença concomitante de rinite alérgica ${ }^{39}$. Em termos clínicos, esta entidade assemelha-se à RO, devendo ser considerada apenas após exclusão definitiva da última através dos procedimentos de diagnóstico indicados. No entanto, o diagnóstico diferencial com a RO nem sempre é possível, sobretudo quando os agentes implicados são agentes de APM, que são também aeroalergénios comuns.

\section{Exame físico}

O exame nasal inclui rinoscopia anterior e endoscopia nasal, sendo sobretudo utilizado para excluir outras patologias nasais. $\mathrm{Na} \mathrm{RO}$ surgem sinais semelhantes aos restantes tipos de rinite, como o edema da mucosa nasal, a hipertrofia dos cornetos inferiores, a rinorreia anterior e/ou posterior. Pode ainda observar-se hemorragia da mucosa na exposição a agentes corrosivos ${ }^{35,36}$.

\section{Testes imunológicos}

Os testes cutâneos por picada (TCP) e/ou o doseamento de anticorpos IgE específicos para os agentes suspeitos permitem demonstrar ou excluir a RO lgE mediada $^{4,36}$. No entanto, no caso dos TCP, os extratos comerciais disponíveis para a maioria dos agentes sensibilizantes de APM e BPM não se encontram estandardizados, e a sua potência alergénica pode variar entre fabricantes ${ }^{40}$. Apesar da potencial utilidade no futuro, não só em termos de diagnóstico, mas também na discriminação entre diferentes vias de exposição (inalação vs contacto com mucosas), o doseamento de lgE específica para componentes moleculares na alergia ocupacional carece ainda de maior investigação ${ }^{40}$. No caso da asma ocupacional, foi documentado um elevado valor preditivo positivo diagnóstico para o látex e para as farinhas de cereais. Vandenplas e col. demonstraram recentemente que a combinação de níveis elevados de slgE para rHev b 5 e Hev b 6,01/6,02 aumentou a especificidade diagnóstica (79\%) e o valor preditivo positivo (>95\%) no diagnóstico de alergia ocupacional ao látex, comparativamente à PPN ${ }^{41}$. Relativamente à alergia 
ocupacional a farinhas de cereais, Sander e col. reportaram que a combinação de slgE para cinco componentes moleculares (Tri a 27, 28, 29.02, 32 e 39) aumentou significativamente a sensibilidade $(70 \%)$ e a especificidade $(97 \%)$ para o diagnóstico de alergia ocupacional a farinha de trigo, enquanto os componentes Tri a 26 e 36 demonstraram importância apenas para o diagnóstico de alergia alimentar ${ }^{42}$. Por outro lado, a presença de Tri a 40.010I (componente molecular do inibidor de alfa-amilase do trigo) não foi relevante para o diagnóstico ${ }^{43}$. Encontram-se atualmente disponíveis outros componentes moleculares para alergénios ocupacionais, como os de origem animal, café, soja, mariscos, porém a sua relevância na sensibilização ocupacional continua por esclarecer ${ }^{40}$.

\section{Prova de provocação nasal}

A PPN através da exposição ao agente ocupacional suspeito é considerada o gold standard para confirmação diagnóstica na $\mathrm{RO}^{4}$. No entanto, a ausência de extratos padronizados limita a capacidade de execução da prova e a interpretação dos resultados.

De acordo com as recomendações da $\mathrm{EAACl}^{44}$, a avaliação das respostas nasais durante a prova pode ser feita de forma subjetiva, utilizando scores de sintomas ou escalas visuais analógicas, e/ou objetiva, analisando a patência nasal. A última poderá ser avaliada através do recurso a um ou mais dos seguintes métodos: pico de fluxo inspiratório nasal (o método mais simples e menos dispendioso para medir o fluxo de ar nasal, embora muito dependente da colaboração do doente), rinomanometria acústica (rápida e fácil de executar, sem necessidade de colaboração do doente), rinometria anterior ativa (atualmente considerado o método standard para avaliação objetiva da patência nasal), rinomanometria de quatro fases (o método tecnicamente mais confiável).

As secreções nasais podem ser colhidas para quantificação da atividade secretora nasal, porém este método carece de validação e estandardização, sendo mais útil em situações em que o indivíduo é comparado consigo próprio durante a PPN ou a avaliação no local de trabalho ${ }^{4}$. A pesquisa de células inflamatórias, sobretudo eosinófilos, e mediadores inflamatórios nas secreções nasais, como a triptase ou a proteína catiónica eosinofílica, também pode ser útill ${ }^{4}$. No caso da rinite alérgica, foi demonstrada uma boa correlação entre a contagem de eosinófilos nas secreções nasais e parâmetros clínicos, incluindo sintomas nasais e patência nasal ${ }^{45}$. O papel do óxido nítrico nasal como biomarcador da inflamação das vias aéreas durante a PPN com agentes ocupacionais ainda não foi bem estabelecido. Em doentes com rinite alérgica, a PPN induziu uma diminuição dos níveis de óxido nítrico, seguida de aumento às $7 \mathrm{~h} e$ 24 h após a prova ${ }^{46}$.

O estudo da hiperreatividade nasal inespecífica na RO não foi ainda bem documentado, contrariamente à prova de hiperreatividade brônquica na asma ${ }^{4}$. Este método permite quantificar de forma inespecífica a hiperreatividade das vias aéreas superiores após a PPN. Perante o estímulo com histamina, metacolina ou ar seco frio, é induzido um aumento da resposta nasal, caracterizado por esternutos, congestão nasal e/ou secreção nasal ${ }^{4,35}$.

$\mathrm{Na}$ ausência de agente identificado ou na presença de vários agentes suspeitos, nas situações em que as condições de exposição no trabalho não podem ser reproduzidas ou em que a PPN não seja exequível, deve considerar-se a realização desta avaliação no local de trabalho ${ }^{4}$. Aqui a resposta nasal é registada antes, durante e após um ou, idealmente, vários turnos de trabalho (para garantir a reprodutibilidade dos resultados) e cada doente é comparado consigo próprio durante um dia de controlo $4,35,36$. Esta estratégia pode ser útil também na investigação da RO induzida por irritantes e na rinite exacerbada pelo trabalho ${ }^{4}$.

\section{Algoritmo diagnóstico}

A Figura I demonstra o algoritmo diagnóstico proposto pela EAACl para a RO (Adaptado da referência 4). 


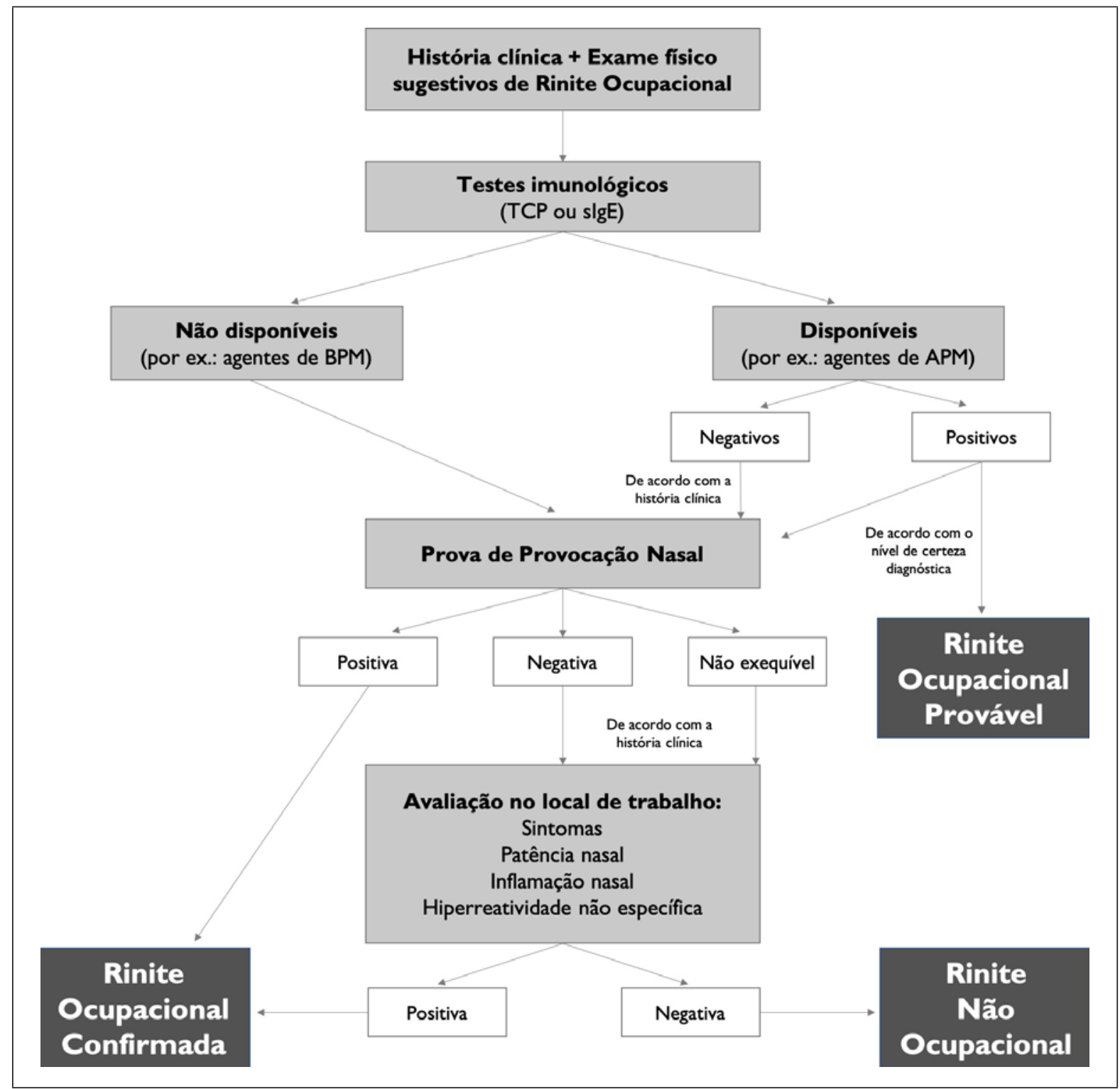

TCP - Testes cutâneos em picada com os agentes suspeitos; slgE - doseamento de anticorpos lgE específicos para os agentes suspeitos; BPM - baixo peso molecular; APM - alto peso molecular

Figura I. Algoritmo diagnóstico para rinite ocupacional de acordo com a $\mathrm{EAACl}^{4}$

\section{ABORDAGEM TERAPÊUTICA}

A abordagem da rinite ocupacional visa dois objetivos principais: minimização dos sintomas nasais e do seu impacto na qualidade de vida do doente e prevenção do desenvolvimento de asma ocupacional. As opções terapêuticas incluem intervenções ambientais destinadas a evitar a exposição ao agente responsável e tratamento farmacológico $4,18,19,47,48$.

\section{Evicção ou redução da exposição}

A opção terapêutica mais segura e eficaz para redução e controlo da RO consiste na identificação do agente 
ocupacional em causa e implementação de medidas de evicção à sua exposição. No entanto, a completa remoção da exposição muitas vezes implica mudanças profissionais consideráveis para os trabalhadores afetados, com consequências socioeconómicas substanciais ${ }^{49,50}$.

A eviç̧ão completa da exposição pode ser implementada com um impacto socioeconómico menor quando existe a possibilidade de recolocação do trabalhador dentro da mesma empresa noutro posto de trabalho sem exposição ${ }^{50,51}$.

A redução significativa da exposição parece ser uma alternativa razoável. De facto, alguns estudos apontam para um benefício clínico da rinite após a redução da exposição aos sais de platina ${ }^{52}$ e ao látex ${ }^{53}$. Esta redução da exposição pode ser conseguida através de diferentes medidas de higiene e segurança como substituição do agente ocupacional causal por outra substância, melhoria das condições de ventilação e exaustão da área de trabalho, redução do tempo de exposição e o uso de equipamentos de proteção individual pelo trabalhador. A formação dos trabalhadores é também eficaz no controlo dos sintomas de rinite ocupacional ${ }^{54,55}$.

Uma recente revisão da Cochrane comparou a evicção total com a diminuição da exposição ao agente causal na asma ocupacional, tendo a primeira apresentado maior eficácia no controlo da asma; no entanto, apresentou um maior risco de desemprego ${ }^{56}$.

\section{Tratamento farmacológico: Farmacoterapia e imunoterapia}

Não existem estudos publicados que avaliem o tratamento farmacológico especificamente na RO, seja alérgica ou não alérgica.

A farmacoterapia não deve ser considerada uma alternativa adequada à eliminação ou redução da exposição no local de trabalho ao agente sensibilizante. No entanto, quando a redução adequada da exposição é impraticável ou insuficiente, a rinite ocupacional deve ser abordada de acordo com as diretrizes e consensos vigentes para rinite não ocupacional ${ }^{47}$.
Para o controlo sintomático recomendam-se agentes farmacológicos, designadamente anti-histamínicos não sedativos, corticoides tópicos nasais, antagonistas dos recetores dos leucotrienos, como em qualquer outra forma de rinite.

Atualmente, não existe evidência científica suficiente para a recomendação da imunoterapia específica na rinite ocupacional. Um número limitado de estudos e relatos de casos isolados documentaram algum benefício relativamente aos sintomas respiratórios durante a imunoterapia com proteínas purificadas de roedores, extratos de farinha de trigo e látex de borracha natural ${ }^{57-59}$. A imunoterapia com alérgenos é atualmente limitada pela indisponibilidade de extratos alergénicos padronizados para a maioria dos agentes ocupacionais ${ }^{57}$. Aguarda-se que as novas tecnologias melhorem a qualidade e eficácia da imunoterapia específica.

\section{Aspetos médico-legais: Avaliação de incapacidade e compensação}

A rinite pode ter um impacto enorme na qualidade de vida do doente e no desempenho laboral. A rinite designa-se como "moderada-grave" sempre que existe compromisso social e/ou laboral ${ }^{15}$.

Atendendo à relevância da RO na epidemiologia da asma ocupacional, parece fundamental implementar medidas de prevenção, nomeadamente a reintegração laboral destes trabalhadores, evitando precocemente a exposição ao agente desencadeante da RO. Torna-se, portanto, importante uma identificação e notificação atempadas da RO.

$\mathrm{Na} \mathrm{RO}$, às dificuldades no diagnóstico e notificação somam-se assim as dificuldades na avaliação da incapacidade e do seu impacto objetivo na atividade laboral desempenhada. A razão deve-se à ausência de critérios objetivos ou valores de referência. A comprovação objetiva da limitação da função nasal é exequível através de estudos de função respiratória nasal (rinomanometria); no entanto, não estão definidas formas de quantificar a gravidade do compromisso nasal na sua globalidade, par- 
ticularmente quando a obstrução nasal não é a condicionante mais relevante.

O grau de gravidade da rinite deve ser determinado de acordo com os sistemas de classificação ${ }^{16}$. A avaliação da incapacidade na asma ocupacional também é problemática, no entanto existe maior experiência nessa área e o grau de gravidade da asma é frequentemente utilizado para determinar a incapacidade associada à doença.

A compensação prática varia entre os diferentes países europeus, prendendo-se com diferenças na definição de RO, avaliação de incapacidade ${ }^{16}$, bem como dos regulamentos e políticas. As doenças profissionais em Portugal constam numa lista organizada e publicada no Diário da República, no Decreto Regulamentar n. ${ }^{\circ}$ 76/2007, de 17 de julho. $\mathrm{Na}$ atual legislação portuguesa a RO não figura como doença profissional.

A reintegração laboral num posto de trabalho sem exposição é recomendada e preferível ao sistema de compensação financeiro ${ }^{26}$.

\section{QUESTÕES POR REVOLVER / PERSPETIVAS FUTURAS}

Um dos grandes desafios na abordagem diagnóstica de RO de causa alérgica prende-se com o diagnóstico diferencial entre esta e a RO não alérgica e a rinite exacerbada pelo trabalho. A evolução no conhecimento sobre a fisiopatologia subjacente a cada uma destas entidades e a sua melhor caraterização clínica deverão permitir uma melhor distinção entre as três.

A incidência e prevalência da RO, ao contrário do que acontece com outras doenças ocupacionais, como a asma, estão mal estabelecidas, o que em parte pode ser explicado pela dificuldade no seu diagnóstico, mas também pelo facto de não ser encarada como uma doença grave. Neste sentido, importa perceber a proporção da rinite na população geral que corresponde a RO; desenvolver e validar questionários que permitam identi- ficar a RO nos estudos epidemiológicos e na prática clínica e sensibilizar os clínicos para esta entidade. É importante também perceber o papel do tabagismo e da atopia no desenvolvimento de RO e esclarecer de que forma os alergénios e agentes irritantes do local de trabalho influenciam a resposta e interação entre as vias aéreas superiores e inferiores.

Para o diagnóstico será importante proceder à padronização de baterias com extratos de alergénios ocupacionais a utilizar nos testes cutâneos por picada, bem como das IgEs específicas a solicitar. É também fundamental a padronização e validação do teste de provocação nasal e das técnicas de medição da hiperreatividade, fluxo e inflamação nasal, estas três últimas sobretudo se usadas para comparar os períodos de exposição ocupacional com os períodos fora do contexto ocupacional (por exemplo, férias ou fins de semana). Por fim, a avaliação do óxido nítrico nasal e a identificação de outros biomarcadores também poderá vir a ser relevante do ponto de vista de diagnóstico.

A avaliação do impacto da $R O$ na qualidade de vida dos doentes e os encargos económicos associados à doença, bem como a resposta a diferentes medidas preventivas e terapêuticas, iriam permitir análises de custo-efetividade que apoiariam a adoção de melhores estratégias na abordagem da RO. No âmbito da prevenção, a identificação de parâmetros que influenciem o prognóstico e o papel do ambiente e das intervenções ambientais na progressão ou controlo da doença são da maior relevância. Outro marco importante na abordagem desta entidade prende-se com análise prospetiva de eficácia e segurança da imunoterapia específica com alergénios ocupacionais no controlo sintomático e na prevenção da progressão para asma ocupacional alérgica.

Por fim, o estabelecimento e uniformização de critérios no que concerne à atribuição de graus de incapacidade decorrentes do impacto da RO no âmbito profissional torna-se também imperativo. 


\section{CONCLUSÃO}

O doente descrito no caso clínico foi submetido a TCP e doseamento de anticorpos IgE específicos (baterias standard, farinhas e cereais, IgE específicas para farinhas, cereais e aeroalergénios) que identificou sensibilização para o trigo, centeio, aveia e glúten. Foi também realizada espirometria com broncodilatação durante o período em exposição, que revelou um padrão obstrutivo a nível das pequenas vias aéreas, que melhorava após o broncodilatador inalado. Complementarmente, foi pedido um diário de registo de sintomas em exposição (atividade laboral) e afastamento (férias e fins de semana), tendo-se verificado a existência de um nexo de causalidade entre exposição ocupacional e a sintomatologia nasal e uma diminuição no PEF > 20\% aquando da exposição ocupacional em comparação com o período de afastamento. Foi estabelecido o diagnóstico de rinite alérgica ocupacional e asma ocupacional e foram dadas recomendações de evicção de exposição profissional, de utilização de equipamento de proteção individual e feita notificação para o departamento de doenças profissionais. Iniciou tratamento com corticoide intranasal, lavagens salinas, anti-histamínico em SOS e broncodilatador de curta duração de ação em SOS. Um ano após o diagnóstico suspendeu atividade laboral, com resolução completa do quadro respiratório superior e inferior, bem como normalização dos parâmetros espirométricos.

\section{Conflito de interesses}

Os autores declaram que não existem conflitos de interesses.

\section{ORCID:}

Cristiana Ferreira (D) 0000-0003-1676-1595

Iolanda Alen (iD) 0000-0002-25II-4843

Maria Marques (iD 0000-0003-224I-7889

Rita Brás (iD) 0000-0002-3059-786X

Ana Margarida Pereira (iD) 0000-0002-5468-0932
Contacto:

Rita Brás

Serviço de Imunoalergologia

Hospital de Santa Maria

Centro Hospitalar Universitário Lisboa Norte

Av. Prof. Egas Moniz

1649-028 Lisboa, Portugal

ritasabras@gmail.com

\section{REFERÊNCIAS}

I. Dykewicz MS, Fineman S, Skoner DP, Nicklas R, Lee R, Blessing-Moore J, et al. Diagnosis and management of rhinitis: Complete guidelines of the Joint Task Force on Practice Parameters in Allergy, Asthma and Immunology. American Academy of Allergy, Asthma, and Immunology. Ann Allergy Asthma Immunol 1998;8I(5 Pt 2):478-518.

2. Grammer LC. Occupational Rhinitis. Immunol Allergy Clin North Am 2016;36(2):333-34I. doi:10.1016/j.iac.2015.12.009

3. Stevens WW, Grammer LC. Occupational rhinitis: an update. Curr Allergy Asthma Rep 2015;I5(I):487. doi:10.1007/s I I882-014-0487-8

4. Moscato G, Vandenplas O, Van Wijk RG, Malo JL, Perfetti L, Quirce $S$, et al. EAACI position paper on occupational rhinitis. Resp Res 2009;10(I):16. doi:10.1186/1465-9921-10-16

5. Garnier R, Villa A, Chataigner D. Rhinites professionnelles. Rev Mal Respir 2007;24(2):205-20. doi:10.1016/S076I-8425(07)91043-8

6. Maoua M, Maalel OE, Kacem I, Guedri S, Ben Kacem M, Aissa S, et al. Quality of life and work productivity impairment of patients with allergic occupational rhinitis. Tanaffos 2019;18(I):58-65.

7. Meggs WJ, Elsheik T, Metzger WJ, Albernaz M, Bloch RM. Nasal pathology and infrastructure in patients with chronic airway inflammation (RADS and RUDS) following an irritant exposure. J Toxicol Clin Toxicol 1996;34(4):383-36. doi:10.3109/155636596090I3808

8. Hoffman CD, Henneberger PK, Olin AC, Mehta A, Torén K. Exposure to ozone gases in pulp mills and the onset of rhinitis. Scand J Work Environ Health 2004;30(6):445-9. doi:10.527I/sjweh.833

9. Moscato G, Pala G, Folletti I, Siracusa A, Quirce S. Occupational rhinitis affects occupational asthma severity. J Occup Health 2016;58(3):310-3.

10. Slavin RG. Update on occupational rhinitis and asthma. Allergy Asthma Proc 2010;3I(6):437-43. doi:10.2500/aap.2010.31.3379

II. Siracusa A, Desrosiers M, Marabini A. Epidemiology of occupational rhinitis: prevalence, aetiology and determinants. Clin Exp Allergy 2000;30(1I):1519-34. doi:10.1046/j.1365-2222.2000. 00946.x

12. Malo JL, Lemière C, Desjardins A, Cartier A. Prevalence and intensity of rhinoconjunctivitis in subjects with occupational asthma. Eur Respir J 1997;10(7):1513-5. doi:10.1183/09031936.97.10071513 
13. Slavin RG. Occupational rhinitis. Ann Allergy Asthma Immunol 2003;90(5 Suppl 2):2-6.

14. Gautrin D, Ghezzo H, Malo J-L. Rhinoconjunctivitis, bronchial responsiveness, and atopy as determinants for incident non-work-related asthma symptoms in apprentices exposed to high-molecular-weight allergens. Allergy 2003;58(7):608-I5. doi:10.1034/j.1398-9995.2003.00197.x

15. Bousquet J, Van Cauwenberge P, Khaltaev N, Aria Workshop Group, World Health Organization. Allergic rhinitis and its impact on asthma. J Allergy Clin Immunol 200I;108(5 Suppl):SI47-334. doi:I0.1067/mai.2001.118891

16. EAACI Task Force on Occupational Rhinitis, Moscato G, Vandenplas O, et al. Occupational rhinitis. Allergy 2008;63(8):969-80. doi:I0.1III/j.1398-9995.2008.0180I.x

17. Malo J-L, Vandenplas $O$. Definitions and classification of work-related asthma. Immunol Allergy Clin North Am 20I I;31 (4):645-62. doi:10.1016/j.iac.2011.07.003

18. Hox V, Steelant B, Fokkens W, Nemery B, Hellings PW. Occupational upper airway disease: how work affects the nose. Allergy 2014;69(3):282-9I. doi:I0.IIII/all.12347

19. Tarlo SM, Lemiere C. Occupational asthma. N Engl J Med 2014;370(7):640-9. doi:10.1056/NEJMral301758

20. Ballal S. Occupational rhinitis revisited: Emphasis on the risk factors in saudi industry. Saudi J Med Med Sci 2016;4(3):154. doi:10.4103/1658-631X.188254

21. Le Moual N, Kauffmann F, Eisen EA, Kennedy SM. The healthy worker effect in asthma: work may cause asthma, but asthma may also influence work. Am J Respir Crit Care Med 2008; 177(I):4-10. doi:10.1164/rccm.200703-4I5PP

22. Jarolímek J, Urban P. Twenty year development of occupational diseases in the Czech Republic: Medical and geographical aspects. Cent Eur J Public Health 2014;22(4):25I-6. doi:10.2110I/cejph. a4063

23. Jang JH, Kim DW, Kim SW, Kim DY, Seong WK, Son TJ, et al. Allergic rhinitis in laboratory animal workers and its risk factors. Ann Allergy Asthma Immunol 2009;102(5):373-7. doi:10.1016/ SI08I-I206(I0)60507-8

24. Moscato G, Siracusa A. Rhinitis guidelines and implications for occupational rhinitis. Curr Opin Allergy Clin Immunol 2009;9(2):II0-5.

25. Hytönen M, Kanerva L, Malmberg H, Martikainen R, Mutanen P, Toikkanen J. The risk of occupational rhinitis. Int Arch Occup Environ Health 1997;69(6):487-90. doi:I0.1007/s004200050I78

26. Nicholson PJ, Cullinan P, Taylor AJN, Burge PS, Boyle C. Evidence based guidelines for the prevention, identification, and management of occupational asthma. Occup Environ Med 2005;62(5):290-9. doi:10.1136/oem.2004.016287

27. Kopferschmitt-Kubler MC, AmeilleJ, Popin E, Calastreng-Crinquand A, Vervloet D, Bayeux-Dunglas MC, et al. Occupational asthma in France: a I-yr report of the observatoire National de Asthmes
Professionnels project. Eur Respir J 2002;19(1):84-9. doi:I0.1/83/0 9031936.02 .00102202

28. Puchner TC, Fink JN. Occupational rhinitis. Immunol Allergy Clin North Am 2000;20(2):303-22. doi:10.1016/S0889-856I(05)70I50-8

29. Yilmaz I, Oner Erkekol F, Secil D, Misirligil Z, Mungan D. Cat and dog sensitization in pet shop workers. Occup Med (Lond) 2013;63(8):563-7. doi:10.1093/occmed/kqtll6

30. Hellings PW, Klimek L, Cingi C, Agache I, Akdis C, Bachert C, et al. Non-allergic rhinitis: Position paper of the European Academy of Allergy and Clinical Immunology. Allergy 20I7;72(II):1657-65. doi: 10.1 III/all.13200

3I. de Meer G, Postma DS, Heederik D. Bronchial responsiveness to adenosine-5'-monophosphate and methacholine as predictors for nasal symptoms due to newly introduced allergens. A follow-up study among laboratory animal workers and bakery apprentices. Clin Exp Allergy 2003;33(6):789-94. doi:10.1046/ j. 1365-2222.2003.01677.x

32. Mapp CE. Genetics and the occupational environment. Curr Opin Allergy Clin Immunol 2005;5(2):II3. doi:I0.1097/0I. all.0000162301.74610.21

33. Nielsen GD, Olsen O, Larsen ST, Løvik M, Poulsen LK, Glue $C$, et al. IgE-mediated sensitisation, rhinitis and asthma from occupational exposures. Smoking as a model for airborne adjuvants? Toxicology 2005;216(2-3):87-105. doi:10.1016/j. tox.2005.07.022

34. Siracusa A, Marabini A, Folletti I, Moscato G. Smoking and occupational asthma. Clin Exp Allergy 2006;36(5):577-84. doi:I0. IIII/ j.1365-2222.2006.02486.x

35. Mungan D. Occupational allergic rhinitis: what do we know? Curr Treat Options Allergy 2015;2(I):10-19. doi:10.1007/s4052I-0I4$-004 \mid-x$

36. Grammer LC. Occupational rhinitis. Immunol Allergy Clin North Am 2016;36(2):333-41. doi:10.1016/j.iac.2015.12.009

37. Karjalainen A, Martikainen R, Klaukka T, Saarinen K, Uitti J. Risk of asthma among finnish patients with occupational rhinitis. Chest 2003;123(I):283-8. doi:10.1378/chest.123.1.283

38. Cortona G, Pisati G, Dellabianca A, Moscato G. [Respiratory occupational allergies: the experience of the Hospital Operative Unit of Occupational Medicine in Lombardy from 1990 to 1998]. G Ital Med Lav Ergon 200I;23(I):64-70. PMID: | |38619|

39. Shusterman D, Murphy MA, Balmes J. Differences in nasal irritant sensitivity by age, gender, and allergic rhinitis status. Int Arch Occup Environ Health 2003;76(8):577-83. doi:10.1007/s00420-003-0459-0

40. Raulf $M$, Quirce $S$, Vandenplas $O$. Addressing molecular diagnosis of occupational allergies. Curr Allergy Asthma Rep 2018;18(I):6. doi:10.1007/s | |882-018-0759-9

4I. Vandenplas O, Froidure A, Meurer U, Rihs HP, Rifflart C, Soetaert $S$, et al. The role of allergen components for the diagnosis of 
latex-induced occupational asthma. Allergy 20I6;7I(6):840-9. doi:I0.IIII/all. 12872

42. Sander I, Rihs HP, Doekes G, Quirce S, Krop E, Rozynek P, et al. Component-resolved diagnosis of baker's allergy based on specific IgE to recombinant wheat flour proteins. J Allergy Clin Immunol 2015;I35(6):I529-37. doi: 10.1016/j.jaci.2014.11.02I

43. Sander I, Rihs H-P, Brüning T, Raulf M. A further wheat allergen for baker's asthma: Tria 40. J Allergy Clin Immunol 2016;137(4):I286. doi:10.1016/j.jaci.2015.11.026

44. Augé J, Vent J, Agache I, Airaksinen L, Campo Mozo P, Chaker A, et al. EAACI Position paper on the standardization of nasal allergen challenges. Allergy 2018;73(8):1597-608. doi:10.1III/all.13416

45. Ciprandi G, Vizzaccaro A, Cirillo I, Tosca M, Massolo A, Passalacqua G: Nasal eosinophils display the best correlation with symptoms, pulmonary function and inflammation in allergic rhinitis. Int Arch Allergy Immunol 2005;136:266-72. doi: 10.1159/000083953

46. Boot JD, de Kam ML, Mascelli MA, Miller B, van Wijk RG, de Groot $\mathrm{H}$, et al. Nasal nitric oxide: Longitudinal reproducibility and the effects of a nasal allergen challenge in patients with allergic rhinitis. Allergy 2007; 62:378-84. doi: 10.1III/j.1398-9995.2007.01328.x

47. Gautrin D, Desrosiers M, Castano R. Occupational rhinitis. Curr Opin Allergy Clin Immunol 2006;6(2):77. doi: 10.1097/0I. all.0000216848.87699.38

48. Moscato G, Pala G, Sastre J. Specific immunotherapy and biological treatments for occupational allergy. Curr Opin Allergy Clin Immunol 2014;I4(6):576-8I. doi: 10.1097/ACI.0000000000000105

49. Moscato G, Dellabianca A, Perfetti L, Bramè B, Galdi E, Niniano $R$, et al. Occupational asthma: a longitudinal study on the clinical and socioeconomic outcome after diagnosis. Chest 1999; II5(I):249-56. doi: 10.1378/chest.II5.1.249

50. Vandenplas O, Toren K, Blanc PD. Health and socioeconomic impact of work-related asthma. Eur Respir J 2003;22(4):689-97. doi: I0.1183/09031936.03.00053203
5I. Piirilä PL, Keskinen HM, Luukkonen R, Salo S-P, Tuppurainen M, Nordman $\mathrm{H}$. Work, unemployment and life satisfaction among patients with diisocyanate induced asthma-A prospective study. J Occup Health 2005;47(2):I12-8. doi: 10.1539/joh.47.II2

52. Merget R, Schulte A, Gebler A, Breitstadt R, Kulzer R, Berndt $E D$, et al. Outcome of occupational asthma due to platinum salts after transferral to low-exposure areas. Int Arch Occup Environ Health 1999;72(I):33-9. doi: 10.1007/s004200050331

53. Vandenplas O, Jamart J, Delwiche J-P, Evrard G, Larbanois A. Occupational asthma caused by natural rubber latex: Outcome according to cessation or reduction of exposure. J Allergy Clin Immunol 2002;109(I):125-30. doi: 10.1067/mai.2002.120760

54. Hellgren J, Karlsson G, Torén K. The dilemma of occupational rhinitis: Management options. Am J Respir Med 2003;2(4):333-4I. doi: 10.1007/BF0325666I

55. Bernstein DI, Karnani R, Biagini RE, Bernstein CK, Murphy K, Berendts $B$, et al. Clinical and occupational outcomes in health care workers with natural rubber latex allergy. Ann Allergy Asthma Immunol 2003;90(2):209-13. doi: 10.1016/sI08I-1206(I0)62I43-6

56. de Groene GJ, Pal TM, Beach J, Beach J, Tarlo SM, Pal TM, et al. Workplace interventions for treatment of occupational asthma. Cochrane Database Syst Rev 201I;(5):CD006308. doi: 10.1002/ |465|858.CD006308.pub3

57. Sastre J, Quirce S. Immunotherapy: an option in the management of occupational asthma? Curr Opin Allergy Clin Immunol 2006;6(2):96-100. doi: 10.1097/0I.all.000021685I.1057I.64

58. Nettis E, Delle Donne P, Di Leo E, Fantini P, Passalacqua G, Bernardini R, et al. Latex immunotherapy: state of the art. Ann Allergy Asthma Immunol 2012;109(3):160-5. doi: 10.1016/j. anai.2012.07.004

59. Hansen I, Hörmann K, Klimek L. [Specific immunotherapy in inhalative allergy to rat epithelium]. Laryngorhinootologie 2004;83(8):512-5. doi: 10.1055/s-2004-8|4505 